\title{
Should chronic hepatitis B mothers breastfeed? a meta analysis
}

Yingjie Zheng ${ }^{1,2^{*}}$, Yihan Lu ${ }^{1,2}$, Qi Ye ${ }^{3}$, Yugang Xia ${ }^{1,2}$, Yueqin Zhou ${ }^{3}$, Qingqing Yao ${ }^{1,2}$ and Shan Wei ${ }^{1,2}$

\begin{abstract}
Background: Hepatitis B virus (HBV) exists in the breast milk of chronic hepatitis B (CHB) mothers. The authors use a meta-analytic technique to quantify the evidence of an association between breastfeeding and risk of CHB infection among the infants vaccinated against HBV.

Methods: Literature search is performed up to 2010 on the relationship between infantile CHB infection within one-year follow up after immunization with the third-dose hepatitis B vaccine and breastfeeding. Two reviewers independently extract the data and evaluate the methodological quality. A random-effects model is employed to systematically combine the results of all included studies.
\end{abstract}

Results: Based on data from 32 studies, 4.32\% (244/5650) of infants born of CHB mothers develop CHB infection. The difference in risk of the infection between breastfed and formula-fed infants (RD) is $-0.8 \%$, (95\% confidence interval [CI]: $-1.6 \%, 0.1 \%)$. Analysis of the data from 16 of the studies finds that RD for mothers who are positive for the HBeAg and/or the HBV DNA, $0.7 \%(95 \% \mathrm{Cl}:-2.0 \%, 3.5 \%)$, is similar to that for those who are negative for these infectivity markers, $-0.5 \%$ (95\%Cl: $-1.7 \%, 0.6 \%)$.

Conclusions: Breast milk is infectious; yet, breastfeeding, even by mothers with high infectivity, is not associated with demonstrable risk of infantile CHB infection, provided that the infants have been vaccinated against HBV at birth.

\section{Background}

The hepatitis B virus (HBV) accounts for a significant morbidity and mortality worldwide. An estimated onethird of the world population has been exposed to HBV and 400 million people are chronic carriers [1]. Mother to infant transmission of $\mathrm{HBV}$ would occur among up to $90 \%$ of infants born of chronic hepatitis B ( CHB) mothers who are positive for the infectivity markers, the $\mathrm{HBeAg}$ and/or the HBV DNA [2]. With the introduction of a safe and effective hepatitis B vaccine, infantile CHB infection has dramatically dropped to around 5\% [3].

The advantages of breastfeeding over formula feeding have been well documented [4]. However, the presence of the HBV in breast milk firstly reported by Linnemann [5] in 1974 and confirmed by later studies [6,7] raised the possibility of transmission of HBV through breastfeeding. But later studies found no association between

\footnotetext{
* Correspondence: yjzheng@shmu.edu.cn

'Department of Epidemiology, School of Public Health, Fudan University, Shanghai 200032, China

Full list of author information is available at the end of the article
}

breastfeeding and infantile CHB infection, both before and after the introduction of hepatitis B vaccine [8-13]. In the absence of evidence that breastfeeding poses any additional risk of infection to infants born of $\mathrm{CHB}$ mothers, World Health Organization (WHO) recommends breastfeeding even for area where HBV infection is highly endemic and HBV vaccine is not available [14].

Scepticism about this recommendation remains among clinicians, however. About $25 \%$ of obstetricians in Australia [15] and 50\% of physicians (most of them were hepatologists!) in Illinois [16] did not recommend CHB mothers to breastfeed their babies. It was noted that $5.4 \%$ of pregnant women in Shanghai were HBsAg positive and $30.3 \%$ of whom were HBeAg-positive (Tao, personal communication), which was reported to pose a significant risk of HBV transmission to infants, including those who had been vaccinated against the virus [17]. In the light of this and other studies [10], it is a common and recommended practice that $\mathrm{CHB}$ mothers with an abnormal alanine aminotransferase (ALT) level before delivery do not breastfeed.
C Biomed Central

() 2011 Zheng et al; licensee BioMed Central Ltd. This is an Open Access article distributed under the terms of the Creative Commons Attribution License (http://creativecommons.org/licenses/by/2.0), which permits unrestricted use, distribution, and reproduction in any medium, provided the original work is properly cited. 
In the present paper, we conduct a meta analysis to assess the risk of $\mathrm{CHB}$ infection of vaccinated infants through breastfeeding associating with $\mathrm{CHB}$ mothers who are and are not positive for the HBV infectivity markers.

\section{Methods}

Data sources and search strategy

Two independent investigators (Ye and Zhou) searched PubMed (1966-), Chinese BioMedical Literature database (CBM, 1978-), Chinese National Knowledge Infrastructure (CNKI, 1979-) and Wanfang Data (Wanfang, 1998-) up to December $31^{\text {th }}, 2010$ by using the combination of MeSH terms "breast feeding" or "breastfeeding" and "hepatitis B". In addition, the reference lists of potentially relevant manuscripts were scanned backward to obtain extra eligible studies.

\section{Criteria for inclusion of the studies}

(1) Follow-up studies, prospective or retrospective, on the association between breastfeeding (BF) versus formula-feeding (FF) and risk of $\mathrm{CHB}$ infection among the infants born of $\mathrm{CHB}$ mothers.

(2) All infants must receive at least 3 doses of hepatitis $B$ vaccines, with or without receiving hepatitis B immunoglobulin (HBIG) after birth.

(3) CHB infection is defined as the presence of any of the three HBV markers (HBsAg, HBeAg and HBV DNA) in blood, during prenatal care visit and/or before delivery of their babies for the mothers and within one year after immunization with the third-dose hepatitis B vaccine for the infants.

\section{Data extraction}

Two independent investigators (Xia and Yao) were involved in data extraction. The third investigator $(\mathrm{Lu})$ examined the results, and a consensus was reached. The outcome, $\mathrm{CHB}$ infection of the infants born of $\mathrm{CHB}$ mothers at the end of follow-up within one year after immunization with the third-dose hepatitis $B$ vaccine was considered. We extracted the following data from the eligible studies: authors' names, journal and year of publication, country of origin, enrolment periods, type of study, number of $\mathrm{CHB}$ and non-CHB among the infants by feeding methods, general characteristics of the babies and their mothers.

\section{Assessment of methodological quality}

Two independent investigators (Yao and Wei) evaluated the quality of each study based on Newcastle-Ottawa Quality Assessment Scale (NOS) [18]. The third investigator (Zheng) examined the results, and a consensus was reached (Table 1). We did not consider the $4^{\text {th }}$ item under selection (Demonstration that outcome of interest was not present at start of study) and the $2^{\text {nd }}$ item under outcome (Was follow-up long enough for outcomes to occur) in NOS, for the reason that the outcomes were available at the end of the follow-up period which was pre-defined in our criteria of inclusion. And we defined the studies with NOS $\geq 5$ as with high quality and the others with NOS $<5$ as with low quality.

\section{Statistical analysis}

The risk difference (RD) with 95\% confidence interval (CI) was used as a measure of effect between breastfeeding (BF) versus formula-feeding (FF) and risk of infantile $\mathrm{CHB}$ infection across studies. The Dersimonian and Laird random-effects model was used to pool the RDs across studies in Stata version 10.0 (Stata Corp). Heterogeneity was explored by $\chi^{2}$ test, with a significance set at a $P$ value $<0.10$. The extent of heterogeneity was measured by Higgins' ${ }^{2}$. Subgroup analysis and Meta-regression were carried out to examine the effect in relation to quality and type of study, language of paper, study sites, hepatitis $B$ vaccination of the mothers and infants, and mothers' infectivity if available. We used the test for interaction to estimate the difference between two subgroups [19]. Publication bias was assessed by visual inspection of a funnel plot, the Egger's and Begger's test [20], and a nonparametric trim and fill method was performed [21].

\section{Results}

1. Literature search

The search retrieved 517 papers, and 99 out of which were potentially relevant to current analysis. Further backward search produced 3 additional papers. Only 32 papers [7,10-13,22-48] which offered 32 independent studies were included in our analysis and the reasons for exclusion of the others were listed in Additional file 1-Figure S1.

\section{General characteristics about the studies included in meta analysis}

The 32 studies included in present analysis were published between 1985 and 2010 (Table 1). One study was conducted in the United States [10], one in Italy [11] and the other 30 in China, including Hongkong. Mothers decided the feeding methods in all studies except one in which they were randomized [38]. The sample size of the studies ranged from 38 to 436 (median, 143), with the ratio of BF versus FF infants of 0.095.07 (median, 0.94).

\section{The risk difference (RD) of infantile CHB infection with methods of feeding}

The 32 studies contributed 5650 infants and $244 \mathrm{CHB}$ outcomes with an overall transmission rate of $4.32 \%$. 
Table 1 General characteristic and quality score of the 32 studies included in this meta analysis

\begin{tabular}{|c|c|c|c|c|c|c|c|c|c|}
\hline No & $\begin{array}{l}\text { Author, } \\
\text { publication } \\
\text { year and } \\
\text { NOS }\end{array}$ & $\begin{array}{l}\text { Mothers } \\
\text { enrolled } \\
\text { period and } \\
\text { sites }\end{array}$ & $\begin{array}{l}\text { Number of } \\
\text { infants, } \\
\text { length of } \\
\text { follow up } \\
\text { and types } \\
\text { of study }\end{array}$ & $\begin{array}{c}\text { Characteristic } \\
\text { of the } \\
\text { mothers }\end{array}$ & $\begin{array}{c}\text { Mothers' } \\
\text { Infectivity } \\
\%\end{array}$ & $\begin{array}{l}\text { Characteristic } \\
\text { of the babies }\end{array}$ & $\begin{array}{c}\text { Infantile } \\
\text { vaccination } *^{2}\end{array}$ & $\begin{array}{l}\text { DBF } \\
(\mathrm{m})\end{array}$ & Other characteristics \\
\hline 1. & $\begin{array}{c}\text { De Martino } \\
{[11]} \\
1985 \\
3\end{array}$ & $\begin{array}{l}\text { NA } \\
\text { Italy }\end{array}$ & $\begin{array}{l}85 \text { cases } \\
12 \text { months } \\
\text { Prospective }\end{array}$ & $\begin{array}{l}\text { APCW, S+ and } \\
\text { C+; a normal } \\
\text { pregnancy; } \\
\text { informed } \\
\text { consent. }\end{array}$ & $2.3(\mathrm{E})$ & $\begin{array}{l}\text { Normal ALT } \\
\text { and birth } \\
\text { weight. }\end{array}$ & $\begin{array}{c}0.5 \mathrm{ml} / \mathrm{kg} \\
\mathrm{HBIG} / 8 \mathrm{~h}+1 \\
\mathrm{ml}(\mathrm{P}) / 2-4-11 \\
\mathrm{~m}\end{array}$ & & $\begin{array}{c}\text { Sex ratio of 1.2, no } \\
\text { medication except a } \\
\text { multivitamin mixture, } \\
\text { vaccination against polio, } \\
\text { tetanus and diphtheria; } \\
\text { monthly visit, no dropout; no } \\
\text { vaccine-related serious side } \\
\text { effect and no abnormal ALT } \\
\text { was observed at the end of } \\
\text { follow up. } \\
\text { Mothers' E+\% and newborns' } \\
\text { gender were similar between } \\
\text { BF and FF. }\end{array}$ \\
\hline 2. & $\begin{array}{c}\text { Tseng [12] } \\
1988 \\
3\end{array}$ & $\begin{array}{l}\text { NA } \\
\text { Hongkong, } \\
\text { China }\end{array}$ & $\begin{array}{c}170 \text { cases } \\
12 \text { months } \\
\text { Retrospective }\end{array}$ & PCW, S+. & $\begin{array}{c}\text { 64(E) } \\
\text { among BF }\end{array}$ & & $\begin{array}{c}0.5 \mathrm{ml} \mathrm{HBIG/} \\
24 \mathrm{~h}+5 \mu \mathrm{g} \\
\text { (P)/0-1-6 m }\end{array}$ & & \\
\hline 3. & $\begin{array}{c}\text { Huang [22] } \\
1993 \\
3\end{array}$ & $\begin{array}{c}\text { Aug. 1989- } \\
\text { Sept. } 1990 \\
\text { Guangdong, } \\
\text { China }\end{array}$ & $\begin{array}{c}112 \text { cases } \\
12 \text { months } \\
\text { Retrospective }\end{array}$ & PCW, S+. & $44.8(\mathrm{E})$ & $\begin{array}{c}\mathrm{S}+\%=6.4 \text { and } \\
\mathrm{E}+\%=25.6(\mathrm{U}) \\
\text { at birth. }\end{array}$ & $\begin{array}{c}30 \mu g(P) / 0-1-6 \\
m\end{array}$ & $9-12$ & $\begin{array}{l}\text { Average age of the mothers } \\
\text { was } 26.97 \pm 2.83 \text { years old. }\end{array}$ \\
\hline 4. & $\begin{array}{c}\text { Wu [23] } \\
1996 \\
3\end{array}$ & $\begin{array}{l}\text { 1992-1993 } \\
\text { Nanjing, } \\
\text { China }\end{array}$ & $\begin{array}{c}80 \text { cases } \\
6 \text { months } \\
\text { Retrospective }\end{array}$ & PCW, S+. & $\begin{array}{l}\text { 55.6(E) } \\
\text { among BF }\end{array}$ & $\begin{array}{c}\text { Full-term } \\
\text { normal delivery. }\end{array}$ & $\begin{array}{c}200 I U \mathrm{HBIG} / 4 \\
\mathrm{~h}, 1 \mathrm{~m}+30 \mu \mathrm{g} \\
\text { (P)/1-3-6 m. }\end{array}$ & 12 & $\begin{array}{l}\text { Mothers' age were similar } \\
\text { between BF and FF. }\end{array}$ \\
\hline 5. & $\begin{array}{c}\text { Zeng [24] } \\
2001 \\
4\end{array}$ & $\begin{array}{c}\text { Jan. 1996- } \\
\text { Jun. } 1999 \\
\text { Guangdong, } \\
\text { China }\end{array}$ & $\begin{array}{c}190 \text { cases } \\
9 \text { months } \\
\text { Retrospective }\end{array}$ & $\begin{array}{l}\text { PCW, S+; } \\
\text { normal ALT. }\end{array}$ & $29.5(\mathrm{E})$ & $\begin{array}{c}\mathrm{S}+\%=14.9(\mathrm{U} \\
\text { 134cases) at } \\
\text { birth. }\end{array}$ & $\begin{array}{c}10 \mu \mathrm{g}(\mathrm{R}) / 0-1- \\
6 \mathrm{~m}\end{array}$ & & $\begin{array}{c}\text { Mothers' } \mathrm{E}+\% \text { was higher in } \\
\text { FF than BF. }\end{array}$ \\
\hline 6. & $\begin{array}{c}\text { Wu [25] } \\
2002 \\
3\end{array}$ & $\begin{array}{l}\text { Jan. 1995- } \\
\text { Dec. } 2000 \\
\text { Nanjing, } \\
\text { China }\end{array}$ & $\begin{array}{c}300 \text { cases } \\
12 \text { months } \\
\text { Retrospective }\end{array}$ & $\begin{array}{c}\text { PCW, S+; } \\
\text { normal hepatic } \\
\text { function; no } \\
\text { nipple crack; } \\
\text { 200IU-HBIG } \\
\text { treatment. }\end{array}$ & $100(E)$ & $\begin{array}{l}\text { Complete } \\
\text { gestation birth; } \\
\text { no oral ulcer. }\end{array}$ & $\begin{array}{c}200 I \cup \text { HBIG/4 } \\
\text { h,1 m+10 } \mu \mathrm{g} \\
\text { (R)/1-3-6 m } \\
\text { with booster. }\end{array}$ & 11 & $\begin{array}{c}\text { Mothers' age range was 25-31 } \\
\text { years. }\end{array}$ \\
\hline 7. & $\begin{array}{c}\text { Hill [10] } \\
2002 \\
6\end{array}$ & $\begin{array}{l}\text { Jan. 1992- } \\
\text { Sept. } 1998 \\
\text { Texas, The } \\
\text { United } \\
\text { States }\end{array}$ & $\begin{array}{l}369 \text { cases } \\
\text { 9-15 months } \\
\text { Prospective }\end{array}$ & $\begin{array}{l}\text { PCW, S+, CG+ } \\
\text { and CM- }\end{array}$ & $\begin{array}{l}24.5(\mathrm{E}, 179 \\
\text { cases) }\end{array}$ & & $\begin{array}{c}0.5 \mathrm{ml} \mathrm{HBIG+} \\
0-1-6 \text { mwith } \\
\text { booster. }\end{array}$ & 4.9 & $\begin{array}{c}\text { Complete follow-up and } \\
\text { vaccination; mothers' } \\
\text { nulliparous\% (all cases), AST/ } \\
\text { ALT level, anti-HCV+\%, HIV-1+ } \\
\%, \text { E+\%, CG+\% and CM+\% } \\
\text { based on available cases, and } \\
\text { newborns' gender were } \\
\text { similar between BF and FF. } \\
\text { Mothers were younger and } \\
\text { with more blacks in FF than } \\
\text { BF. }\end{array}$ \\
\hline 8. & $\begin{array}{c}\text { Wang [13] } \\
2003 \\
5\end{array}$ & $\begin{array}{c}\text { 1994-1999 } \\
\text { Shanghai, } \\
\text { China }\end{array}$ & $\begin{array}{c}230 \text { cases } \\
12 \text { months } \\
\text { Retrospective }\end{array}$ & $\begin{array}{l}\text { APCW, S+; } \\
\text { normal ALT. }\end{array}$ & $34(E)$ & $\begin{array}{c}37-42 \text { gestation } \\
\text { weeks; } \mathrm{S}+\%= \\
10.9 \text { at birth. }\end{array}$ & $\begin{array}{c}30 \mu \mathrm{g}(\mathrm{P}, \\
\text { before } 1997) \\
\text { or } 5 \mu \mathrm{g}(\mathrm{R}, \\
\text { after } 1997) / 0- \\
1-6 \mathrm{~m} \text { or/24 h } \\
+1-2-7 \mathrm{~m}\end{array}$ & $\geq 2$ & $\begin{array}{c}\text { Those infants were excluded } \\
\text { with an obvious abnormality, } \\
\text { birth weight }<2500 \mathrm{~g} \text {, or an } \\
\text { apgar score of }<8 \text { at } 1 \text { or } 5 \\
\text { minutes. } \\
\text { Mothers' E+\%, newborns' } \\
\text { gender, birth weight and } \mathrm{S}+\% \\
\text { at birth were similar between } \\
\text { BF and FF. } \\
\text { BF group received more } \\
\text { active immunization than FF. }\end{array}$ \\
\hline 9. & $\begin{array}{c}\text { Zeng [26] } \\
2003 \\
2\end{array}$ & $\begin{array}{l}\text { May 1995- } \\
\text { Sept. } 1997 \\
\text { Sichuang, } \\
\text { China }\end{array}$ & $\begin{array}{c}53 \text { cases } \\
12 \text { months } \\
\text { Retrospective }\end{array}$ & PCW, S+. & $\begin{array}{l}100(E \text { or } \\
\text { DNA) }\end{array}$ & S-at birth. & $\begin{array}{c}200 \mathrm{IU} \mathrm{HBIG} / \\
24 \mathrm{~h}+30 \mu \mathrm{g} \\
(\mathrm{P}) / 2 \mathrm{w}, 10 \mu \mathrm{g} / \\
1.5 \mathrm{~m}, 10 \mu \mathrm{g} / \\
6.5 \mathrm{~m}\end{array}$ & $\geq 4$ & $\begin{array}{c}\text { Duration of breastfeeding }<4 \\
\text { months was excluded. }\end{array}$ \\
\hline
\end{tabular}


Table 1 General characteristic and quality score of the 32 studies included in this meta analysis (Continued)

\begin{tabular}{|c|c|c|c|c|c|c|c|c|c|}
\hline$\overline{10 .}$ & $\begin{array}{c}\text { Gu [27] } \\
2003 \\
3\end{array}$ & $\begin{array}{l}\text { 1998-1999 } \\
\text { Nanjing, } \\
\text { China }\end{array}$ & $\begin{array}{l}152 \text { cases } \\
12 \text { months } \\
\text { Prospective }\end{array}$ & $\begin{array}{c}\text { PCW, S+ and C } \\
+; 200 \sim 400 I U- \\
\text { HBIG } \\
\text { treatment }\end{array}$ & $O(E)$ & $\begin{array}{c}\text { Healthy; } \\
\text { complete } \\
\text { gestation } \\
\text { weeks; birth } \\
\text { weight }>3000 \\
\text { g; } \text { S+\% }=7.6 \text { at } \\
\text { birth. }\end{array}$ & $\begin{array}{c}10 \mu g(R, 80 \\
\text { cases) or } 30 \\
\mu g(P, 72 \\
\text { cases }) / 24 \text { h- } 1- \\
6 \mathrm{~m}\end{array}$ & & $\begin{array}{c}\text { Proportion of types of } \\
\text { vaccines was similar between } \\
\text { BF and FF. }\end{array}$ \\
\hline 11. & $\begin{array}{c}\mathrm{He}[28] \\
2004 \\
3\end{array}$ & $\begin{array}{l}\text { Dec. } 1997- \\
\text { Dec. } 1999 \\
\text { Inner } \\
\text { Mongolia, } \\
\text { China }\end{array}$ & $\begin{array}{l}38 \text { cases } \\
12 \text { months } \\
\text { Retrospective }\end{array}$ & PCW, S+. & 100(DNA) & $\begin{array}{c}\mathrm{DNA}+\%=56 \\
(\mathrm{U})\end{array}$ & $\begin{array}{l}\text { 100-200IU } \\
\mathrm{HBIG} / 6 \mathrm{~h}, 2 \mathrm{w} \\
+0-1-6 \mathrm{~m}\end{array}$ & & \\
\hline 12. & $\begin{array}{l}\text { Liu [29] } \\
2004 \\
3\end{array}$ & $\begin{array}{l}\text { Feb. 1998- } \\
\text { Feb. } 2001 \\
\text { Henan, } \\
\text { China }\end{array}$ & $\begin{array}{c}436 \text { cases } \\
12 \text { months } \\
\text { Retrospective }\end{array}$ & $\begin{array}{l}\text { APCW, S+; } \\
\text { 200IU-HBIG } \\
\text { treatment. }\end{array}$ & & $\begin{array}{c}\mathrm{S}+\%=8.7 \text { and } \\
\mathrm{E}+\%=0.9 \text { at } \\
\text { birth }\end{array}$ & $\begin{array}{c}200 \mathrm{U} \text { HBIG/ } \\
24 \mathrm{~h}, 2 \mathrm{w}+5 \\
\mu \mathrm{g}(\mathrm{R}) / 0-1-6 \mathrm{~m}\end{array}$ & & $\begin{array}{c}\text { Newborns' } \mathrm{S}+\% \text { and } \mathrm{E}+\% \text { at } \\
\text { birth were similar between BF } \\
\text { and } \mathrm{FF} \text {. }\end{array}$ \\
\hline 13. & $\begin{array}{c}\text { Yao [30] } \\
2004 \\
4\end{array}$ & $\begin{array}{l}\text { Dec. 2000- } \\
\text { Jun. } 2002 \\
\text { Zhejiang, } \\
\text { China }\end{array}$ & $\begin{array}{c}190 \text { cases } \\
12 \text { months } \\
\text { Retrospective }\end{array}$ & $\begin{array}{l}\text { PCW, S+; } \\
\text { 200IU-HBIG } \\
\text { treatment. }\end{array}$ & $72.9(\mathrm{E})$ & $\begin{array}{c}\text { Healthy; } \\
\text { complete } \\
\text { gestation } \\
\text { weeks; DNA+\% } \\
=5.0 \text { and } \mathrm{S}+\% \\
=6.3 \text { at birth. }\end{array}$ & $\begin{array}{l}200 I \cup \mathrm{HBIG} / 6- \\
12 \mathrm{~h}, 2 \mathrm{w}+5 \\
\mu g(R) / 0-1-6 \mathrm{~m}\end{array}$ & & $\begin{array}{c}\text { Mothers' age, uterine } \\
\text { contraction+\%, duration of } \\
\text { uterine contraction, E+\% and } \\
\text { HBV+\%, and newborns' } \\
\text { gestation weeks and birth } \\
\text { weight were similar between } \\
\text { BF and FF. }\end{array}$ \\
\hline 14. & $\begin{array}{c}\text { Meng }[31] \\
2004 \\
4\end{array}$ & $\begin{array}{l}\text { Mar. 2001- } \\
\text { Mar. } 2003 \\
\text { Guangdong, } \\
\text { China }\end{array}$ & $\begin{array}{l}134 \text { cases } \\
6 \text { months } \\
\text { Prospective }\end{array}$ & $\begin{array}{l}\text { PCW, S+; } \\
\text { normal ALT; } \\
\text { 200IU-HBIG } \\
\text { treatment (66 } \\
\text { cases). }\end{array}$ & & $\begin{array}{c}\mathrm{S}+\%=16.4, \mathrm{E}+ \\
\%=6.7 \text { and } \\
\text { DNA } \%=2.2 \\
\text { at birth. }\end{array}$ & $0-1-6 \mathrm{~m}$ & & $\begin{array}{l}\text { No foetus abnormality, no } \\
\text { history of treatment of anti- } \\
\text { virus drugs during pregnancy, } \\
\text { TORCH syndrome and other } \\
\text { diseases. }\end{array}$ \\
\hline 15. & $\begin{array}{l}\text { Mou [32] } \\
2005 \\
5\end{array}$ & $\begin{array}{l}\text { Sept. } 2001- \\
\text { Oct. } 2003 \\
\text { Shandong, } \\
\text { China }\end{array}$ & $\begin{array}{c}91 \text { cases } \\
12 \text { months } \\
\text { Prospective }\end{array}$ & $\begin{array}{l}\text { PCW, S+; } \\
\text { normal hepatic } \\
\text { function; } \\
\text { informed } \\
\text { consent. }\end{array}$ & $\begin{array}{c}\text { 33.0(E) } \\
\text { 37.4(DNA) }\end{array}$ & $\begin{array}{l}\text { DNA }+\%=13.2 \\
\text { (U) at birth. }\end{array}$ & $\begin{array}{l}200 I \cup \text { HBIG/ } \\
12 \text { h,2 w+0-1- } \\
6 \text { m with } \\
\text { booster. }\end{array}$ & $\geq 1$ & $\begin{array}{c}\text { Exclusion for those with } \\
\text { premature birth, low birth } \\
\text { weight, asphyxia and history } \\
\text { of bleeding during } \\
\text { pregnancy, breastfeed < } 1 \\
\text { month. } \\
\text { Mothers' E+\% and DNA+\%, } \\
\text { newborns' gestation weeks, } \\
\text { birth weight and DNA+\% (U) } \\
\text { were similar between BF and } \\
\text { FF. }\end{array}$ \\
\hline 16. & $\begin{array}{c}\text { Zhang [33] } \\
2005 \\
4\end{array}$ & $\begin{array}{l}\text { 2000-2004 } \\
\text { Ningxia, } \\
\text { China }\end{array}$ & $\begin{array}{l}104 \text { cases } \\
12 \text { months } \\
\text { Prospective }\end{array}$ & $\begin{array}{l}\text { PCW, S+; } \\
\text { 200IU-HBIG } \\
\text { treatment. }\end{array}$ & $100(E)$ & & $\begin{array}{c}200 \mathrm{U} \text { HBIG/ } \\
24 \mathrm{~h}, 1 \mathrm{~m}+10 \\
\mu \mathrm{g}(\mathrm{R}) / 2-3-6 \mathrm{~m}\end{array}$ & & Natural delivery $\%=50.0$ \\
\hline 17. & $\begin{array}{c}\text { Zhang [34] } \\
2005 \\
3\end{array}$ & $\begin{array}{l}\text { Jan. } 2001- \\
\text { Feb. } 2002 \\
\text { Chongqing, } \\
\text { China }\end{array}$ & $\begin{array}{c}67 \text { cases } \\
12-18 \\
\text { months } \\
\text { Retrospective }\end{array}$ & $\begin{array}{l}\text { PCW, S+; } \\
\text { 100IU-HBIG } \\
\text { treatment. }\end{array}$ & $28.4(E)$ & & $\begin{array}{l}200 I U \text { HBIG/ } \\
24 \mathrm{~h}+100 I \mathrm{U} \\
\mathrm{HBIG} / 15 \mathrm{~d}+10 \\
\mu \mathrm{g}(\mathrm{R}) / 2-3-6 \mathrm{~m}\end{array}$ & & $\begin{array}{c}\text { Mothers' E+\% was higher in } \\
\text { FF than BF. }\end{array}$ \\
\hline 18. & $\begin{array}{l}\text { Qiu [35] } \\
2005 \\
3\end{array}$ & $\begin{array}{l}\text { Dec. 2002- } \\
\text { Mar. } 2004 \\
\text { Zhejiang, } \\
\text { China }\end{array}$ & $\begin{array}{c}90 \text { cases } \\
6 \text { months } \\
\text { Retrospective }\end{array}$ & $\begin{array}{c}\text { PCW, S+; } \\
\text { normal hepatic } \\
\text { function; no } \\
\text { nipple crack; } \\
\text { 200IU-HBIG } \\
\text { treatment. }\end{array}$ & $100(E)$ & $\begin{array}{l}\text { Healthy; } \\
\text { complete } \\
\text { gestation } \\
\text { weeks. }\end{array}$ & $\begin{array}{c}200 \mathrm{U} \mathrm{HBIG} / 6 \\
\mathrm{~h}+5 \mathrm{\mu g}(\mathrm{R}) / 12 \\
\mathrm{~h}-1-6 \mathrm{~m}\end{array}$ & $>6$ & $\begin{array}{l}\text { Mothers' age and DNA+\%, } \\
\text { newborns' gestation weeks } \\
\text { were similar between BF and } \\
\text { FF. }\end{array}$ \\
\hline 19. & $\begin{array}{c}\text { Zeng [36] } \\
2006 \\
4\end{array}$ & $\begin{array}{l}\text { Jun. } 2001- \\
\text { Dec. } 2003 \\
\text { Guangxi, } \\
\text { China }\end{array}$ & $\begin{array}{c}115 \text { cases } \\
7 \text { months } \\
\text { Prospective }\end{array}$ & $\begin{array}{l}\text { PCW, S+; } \\
\text { normal ALT; } \\
\text { 200IU-HBIG } \\
\text { treatment. }\end{array}$ & 31.3(E) & $\begin{array}{c}\text { Apgar }>7 ; \\
\text { birth weight }> \\
2500 \mathrm{~g} ; \mathrm{S}+\%= \\
12.2 \text { at birth. }\end{array}$ & $\begin{array}{l}200 I \mathrm{U} \text { HBIG/ } \\
24 \mathrm{~h}, 1 \mathrm{~m}+5 \\
\mu \mathrm{g}(\mathrm{R}) / 72 \mathrm{~h}-1- \\
6 \mathrm{~m}\end{array}$ & $>0.5$ & $\begin{array}{c}\text { Mothers' E+\%, newborns' } \\
\text { gender, birth weight and HBV } \\
\text { +\% at birth were similar } \\
\text { between BF and FF. }\end{array}$ \\
\hline 20. & $\begin{array}{c}\text { Ma [7] } \\
2006 \\
4\end{array}$ & $\begin{array}{l}\text { 1993-1999 } \\
\text { Liaoning, } \\
\text { China }\end{array}$ & $\begin{array}{c}167 \text { cases } \\
9 \text { months } \\
\text { Retrospective }\end{array}$ & $\mathrm{PCW}, \mathrm{S}+$. & $54(E)$ & $\begin{array}{c}\text { Birth } \\
\text { temperature < } \\
37^{\circ} \mathrm{C} \text {; birth } \\
\text { weight }>2500 \\
\text { g; apgar }>8 .\end{array}$ & (R)/0-1-6 m & & $\begin{array}{l}\text { Mothers' } \mathrm{E}+\% \text { was similar } \\
\text { between BF and FF. }\end{array}$ \\
\hline
\end{tabular}


Table 1 General characteristic and quality score of the 32 studies included in this meta analysis (Continued)

\begin{tabular}{|c|c|c|c|c|c|c|c|c|c|}
\hline 21. & $\begin{array}{l}\text { Chen [37] } \\
2006 \\
4\end{array}$ & $\begin{array}{l}\text { Mar. 2002- } \\
\text { May. 2005 } \\
\text { Zhejiang, } \\
\text { China }\end{array}$ & $\begin{array}{l}170 \text { cases } \\
12 \text { months } \\
\text { Retrospective }\end{array}$ & $\begin{array}{l}\text { APCW, S+; } \\
\text { normal hepatic } \\
\text { function; } \\
\text { informed } \\
\text { consent. }\end{array}$ & $41.2(\mathrm{E})$ & $\begin{array}{c}\mathrm{DNA}+\%=9.4 \\
(U) .\end{array}$ & $\begin{array}{l}200 \mid \cup \text { HBIG/ } \\
12 \mathrm{~h}, 14 \mathrm{~d}+5 \\
\mu \mathrm{g}(\mathrm{R}) / 0-1-6 \\
\text { m with } \\
\text { booster }\end{array}$ & $\geq 1$ & $\begin{array}{c}\text { Exclusion for those with } \\
\text { premature birth, low birth } \\
\text { weight, asphyxia and history } \\
\text { of bleeding during } \\
\text { pregnancy, breastfeeding } \\
\text { below one month. } \\
\text { Mothers' E+\% and DNA+\%, } \\
\text { newborns' birth weight, } \\
\text { gestation weeks and DNA+\% } \\
\text { were similar between BF and } \\
\text { FF. }\end{array}$ \\
\hline 22. & $\begin{array}{l}\text { Zuo [38] } \\
2007 \\
4\end{array}$ & $\begin{array}{l}\text { May 2004- } \\
\text { May 2005 } \\
\text { Hebei, China }\end{array}$ & $\begin{array}{l}180 \text { cases } \\
12 \text { months } \\
\text { prospective }\end{array}$ & $\begin{array}{l}\text { PCW, S+; } \\
\text { 200IU-HBIG } \\
\text { treatment. }\end{array}$ & 49.4(E) & $\begin{array}{c}\mathrm{S}+\%=6.7 \text { and } \\
\text { DNA }+\%=4.2 \\
\text { at birth. }\end{array}$ & $\begin{array}{c}200 \mathrm{IU} \mathrm{HBIG} / \\
48 \mathrm{~h}, 1 \mathrm{~m}, 3 \mathrm{~m} \\
+5 \mu \mathrm{g}(\mathrm{R}) / 24 \\
\mathrm{~h}-1-6 \mathrm{~m}\end{array}$ & 10 & $\begin{array}{c}\text { The infants were randomized } \\
\text { into two groups. } \\
\text { Mothers' age, E+\% and DNA+ } \\
\% \text {, newborns' birth weight, } \\
\text { method of delivery, S+\% and } \\
\text { DNA+\% were similar between } \\
\text { BF and FF. }\end{array}$ \\
\hline 23. & $\begin{array}{l}\text { Wang [39] } \\
2007 \\
3\end{array}$ & $\begin{array}{l}\text { Oct. 2003- } \\
\text { Jan. } 2006 \\
\text { Ningxia, } \\
\text { China }\end{array}$ & $\begin{array}{c}165 \text { cases } \\
10-12 \\
\text { months } \\
\text { Retrospective }\end{array}$ & APCW, S+. & $O(E)$ & $\begin{array}{c}\mathrm{S}+\%=11.5 \\
\text { and } \mathrm{E}+\%=2.4 .\end{array}$ & $\begin{array}{c}200 I \cup \mathrm{HBIG} / 6 \\
\mathrm{~h}+5 \mu \mathrm{g}(\mathrm{R}) / 2 \\
\mathrm{~d}-1-6 \mathrm{~m}\end{array}$ & & $\begin{array}{l}\text { Mothers' age and DNA level, } \\
\text { newborns' sex ratio, birth } \\
\text { weight and method of } \\
\text { delivery were similar between } \\
\text { BF and FF. }\end{array}$ \\
\hline 24. & $\begin{array}{l}\text { Qin [40] } \\
2007 \\
3\end{array}$ & $\begin{array}{l}\text { Jan. 2001- } \\
\text { Jan. } 2005 \\
\text { Henan, } \\
\text { China }\end{array}$ & $\begin{array}{c}74 \text { cases } \\
12 \text { months } \\
\text { Retrospective }\end{array}$ & $\begin{array}{l}\text { PCW, DNAt; } \\
\text { 200IU-HBIG } \\
\text { treatment. }\end{array}$ & $100(\mathrm{DNA})$ & $\begin{array}{l}\mathrm{DNA}+\%=4.5 \\
\text { at birth }\end{array}$ & $\begin{array}{c}200 \mathrm{IU} \mathrm{HBIG/} \\
16 \mathrm{~h}+10 \mu \mathrm{g} \\
(\mathrm{R}) / 1-2-7 \mathrm{~m} \text { or } \\
10 \mu \mathrm{g}(\mathrm{R}) / 0-1-6 \\
\mathrm{~m}\end{array}$ & & \\
\hline 25. & $\begin{array}{c}\text { Chen [41] } \\
2008 \\
4\end{array}$ & $\begin{array}{l}\text { Oct. 2002- } \\
\text { Aug. } 2007 \\
\text { Beijing, } \\
\text { China }\end{array}$ & $\begin{array}{c}345 \text { cases } \\
12 \text { months } \\
\text { Retrospective }\end{array}$ & PCW, S+. & $\begin{array}{l}0(E \text { and } \\
\text { DNA) }\end{array}$ & & $\begin{array}{l}10 \mu g(R) / 0-1-6 \\
m+100-200 I U \\
H B I G / 24 h \\
15-30 d(241 \\
\text { cases). }\end{array}$ & & $\begin{array}{l}\text { Proportion of infants who } \\
\text { received HBIG was higher in } \\
\text { FF than BF. }\end{array}$ \\
\hline 26. & $\begin{array}{l}\text { Hou [42] } \\
2009 \\
4\end{array}$ & $\begin{array}{l}\text { Jan. 2002-NA } \\
\text { Shenzhen, } \\
\text { China }\end{array}$ & $\begin{array}{l}369 \text { cases } \\
9-15 \text { months } \\
\text { Retrospective }\end{array}$ & $\begin{array}{c}\text { PCW, S+; } \\
\text { abnormal ALT/ } \\
\text { AST\% }=2.2 .\end{array}$ & 13.6(E) & & $\begin{array}{c}\text { 100IU HBIG/1 } \\
\mathrm{d}+5 \mu \mathrm{g}(\mathrm{R}) / 0- \\
\text { 1-6 m with } \\
\text { booster. }\end{array}$ & 4.9 & $\begin{array}{l}\text { Mothers' age, abnormal ALT/ } \\
\text { AST\%, E+\%, CM+\% and CG+ } \\
\%, \text { newborns' sex ratio were } \\
\text { similar between BF and FF. }\end{array}$ \\
\hline 27. & $\begin{array}{l}\mathrm{Li}[43] \\
2009 \\
2\end{array}$ & $\begin{array}{l}\text { 2004-2007 } \\
\text { Shandong, } \\
\text { China }\end{array}$ & $\begin{array}{c}45 \text { cases } \\
12 \text { months } \\
\text { Retrospective }\end{array}$ & $\begin{array}{l}\text { APCW, S+; } \\
\text { normal hepatic } \\
\text { function; } \\
\text { 20OIU-HBIG } \\
\text { treatment. }\end{array}$ & 33.3(E) & $\begin{array}{l}\mathrm{DNA}+\%=10.0 \\
\text { at birth. }\end{array}$ & $\begin{array}{l}200 \text { IU HBIG/ } \\
12 \text { h, } 2 \text { w+ 0- } \\
1-6 \text { m with } \\
\text { booster. }\end{array}$ & & $\begin{array}{c}\text { Mothers' E+\% and DNA+\%, } \\
\text { newborns' gestation weeks, } \\
\text { birth weight and DNA+\% } \\
\text { were similar between BF and } \\
\text { FF. }\end{array}$ \\
\hline 28. & $\begin{array}{l}\text { Luo [44] } \\
2010 \\
4\end{array}$ & $\begin{array}{l}\text { 2007-2009 } \\
\text { Fujian, China }\end{array}$ & $\begin{array}{l}436 \text { cases } \\
6 \text { months } \\
\text { retrospective }\end{array}$ & $\begin{array}{l}\text { PCW, S+; } \\
\text { normal hepatic } \\
\text { function; } \\
\text { 20OIU-HBIG } \\
\text { treatment. }\end{array}$ & 44.0(E) & & $\begin{array}{l}200 \mathrm{IU} \mathrm{HBIG} / \\
12 \mathrm{~h}, 2 \mathrm{w}+5 \\
\mu \mathrm{g}(\mathrm{R}) / 0-1-6 \mathrm{~m}\end{array}$ & $>6$ & $\begin{array}{l}\text { Mothers' } \mathrm{E}+\% \text { and } \mathrm{DNA}+\% \\
\text { were higher in FF than BF. }\end{array}$ \\
\hline 29. & $\begin{array}{c}\text { Chen [45] } \\
2010 \\
3\end{array}$ & $\begin{array}{l}\text { 2001-2009 } \\
\text { Shandong, } \\
\text { China }\end{array}$ & $\begin{array}{l}278 \text { cases } \\
8-12 \text { months } \\
\text { Retrospective }\end{array}$ & $\begin{array}{l}\text { PCW, S+; } \\
\text { 20OIU-HBIG } \\
\text { voluntary } \\
\text { treatment. }\end{array}$ & & & $\begin{array}{l}200 I U \text { HBIG/ } \\
24 \text { h,2 w or } \\
200 I U \text { HBIG/ } \\
24 \text { h }+0-1-6 \\
\text { m or 0-1-2-7 } \\
\text { m }\end{array}$ & & $\begin{array}{c}\text { Mothers' age range was 21-40 } \\
\text { years old. }\end{array}$ \\
\hline 30. & $\begin{array}{c}\text { Sun }[46] \\
2010 \\
2\end{array}$ & $\begin{array}{l}\text { Nov. 2007- } \\
\text { Jul. } 2008 \\
\text { Shandong, } \\
\text { China }\end{array}$ & $\begin{array}{l}69 \text { cases } \\
12 \text { months } \\
\text { retrospective }\end{array}$ & PCW, S+. & & DNA-at birth & $\begin{array}{c}\mathrm{HBIG}+(\mathrm{R}) / 0-1- \\
6 \mathrm{~m}\end{array}$ & & \\
\hline 31. & $\begin{array}{l}\text { Liu [47] } \\
2010 \\
4\end{array}$ & $\begin{array}{l}\text { Jan. 2009- } \\
\text { Mar. } 2010 \\
\text { Guangdong, } \\
\text { China }\end{array}$ & $\begin{array}{l}145 \text { cases } \\
12 \text { months } \\
\text { retrospective }\end{array}$ & $\begin{array}{l}\text { PCW, S+; } \\
\text { 200IU-HBIG } \\
\text { treatment. }\end{array}$ & $58.1(\mathrm{DNA})$ & & $\begin{array}{c}200 I \cup \text { HBIG }+5 \\
\mu \mathrm{g}(\mathrm{R}) / 0-1-6 \\
\mathrm{~m}\end{array}$ & $>6$ & $\begin{array}{l}\text { Mothers' DNA+\%, age, time } \\
\text { of pregnancy and delivery, } \\
\text { methods of delivery, } \\
\text { newborns' birth weight, } S+\% \\
\text { and DNA+\% at birth were } \\
\text { similar between BF and FF. }\end{array}$ \\
\hline
\end{tabular}


Table 1 General characteristic and quality score of the 32 studies included in this meta analysis (Continued)

\begin{tabular}{|c|c|c|c|c|c|c|c|}
\hline 32. & $\begin{array}{c}\text { Wu [48] } \\
2010 \\
3\end{array}$ & $\begin{array}{l}\text { Sept. 2007- } \\
\text { May. } 2009 \\
\text { Guangdong, } \\
\text { China }\end{array}$ & $\begin{array}{c}46 \text { cases } \\
12 \text { months } \\
\text { Retrospective }\end{array}$ & $\mathrm{PCW}, \mathrm{S}+$. & $\begin{array}{l}100 \text { (both } \\
\text { E and } \\
\text { DNA) }\end{array}$ & $\begin{array}{c}200 I \cup \mathrm{HBIG} / 6 \\
\mathrm{~h}+5 \mu \mathrm{g}(\mathrm{R}) / 0- \\
1-6 \mathrm{~m}\end{array}$ & $\begin{array}{c}\text { Mothers' age, newborns' sex } \\
\text { ratio, birth weight and } \\
\text { methods of delivery were } \\
\text { similar between BF and FF. }\end{array}$ \\
\hline
\end{tabular}

NOS: Newcastle-Ottawa Quality Assessment Scale; NA: not available; BF: breastfeeding; FF: formula feeding; hepatitis B immunoglobulin: HBIG; APCW: asymptomatic prenatal care women; PCW: prenatal care women; S: HBsAg; E: HBeAg; C: HBcAb; CG: HBcAb IgG; CM: HBcAb IgM; DNA: HBV DNA; P: plasmaderived vaccine; R: recombinant vaccine; U: umbilical cord blood; ALT: alanine aminotransferase; AST: aspartate aminotransferase; IU: international unit; m: month; w: week; d: day; h: hours; ml: millilitre; kg: kilogram; $\mu \mathrm{g}$ : microgram; DBF: duration of breastfeeding

${ }^{* 1}$ : HBIG treatment: HBIG was received by the mothers at the gestation weeks of 28 th, 32 th and 36 th each.

$*^{2}$ : One boost dose was given to those infants with negative HBsAb status.

The difference between the risk of infantile $\mathrm{CHB}$ infection among BF and FF infants (RD) determined by the random-effects model was $-0.8 \%$, $(95 \%$ confidence interval $[\mathrm{CI}]:-1.6 \%, 0.1 \%$ ) (Figure 1 ) and that determined by the fixed-effects model was $-0.4 \%$ (95\% CI: $-1.6 \%, 0.7 \%)$. The findings suggest that $\mathrm{BF}$ is not associated with additional risk of infantile $\mathrm{CHB}$ infection concurred with that of all the individual studies, except one [11], which suggests that $\mathrm{BF}$ is associated with a lower risk than FF.

Subgroup studies showed that the RDs could be modified by language of paper and quality of study, but not

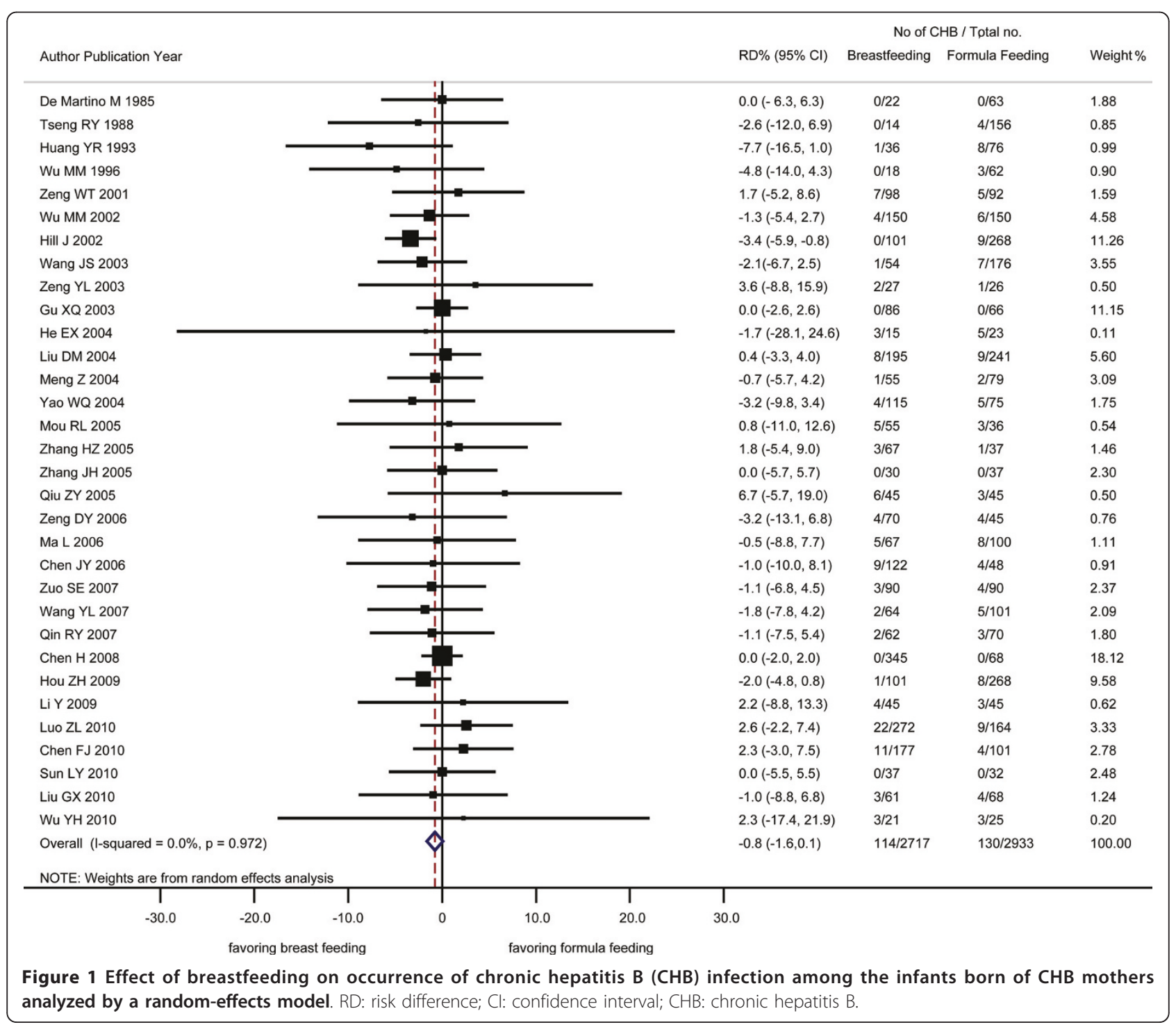


by type of study, study sites or infant-mother vaccination. The studies in English or with high quality tended to report lower RDs, which would probably reflect the difference of mothers' HBV infectivity (Table 2).

Data on HBV infectivity available from 16 of the 32 studies showed that $42.0 \%$ (1648/3924) of mothers were designated with "high infectivity", being positive for either the HBeAg and/or the HBV DNA, and that the proportion of these mothers was lower among those who elected to BF $(36.9 \%, 739 / 2001)$ than those who elected to FF $(44.1 \%, 812 / 1843)$ (Table 2). The overall RD was $-0.4 \%$ (95\% CI: $-1.4 \%, 0.7 \%$ ), which was not modified by language of paper, quality and type of study, study sites or infant-mother vaccination by both subgroup analysis and Meta regression. And the RD determined for mothers with high infectivity was $0.7 \%$ (95\% CI: $-2.0 \%$, $3.5 \%)$, which was not significantly different $(\mathrm{Z}=0.789, P$ $>0.05$ ) from that determined for mothers with low infectivity, $-0.5 \%$ (95\% CI: $-1.7 \%, 0.6 \%$ ) (Figure 2).

\section{Publication bias and its correction}

Visual inspection of the funnel plots demonstrated a possible publication bias. After trim and fill methods were performed, the pooled RDs were $-1.0 \%$ (95\%CI: $-1.8 \%,-0.1 \%)$ and $-0.6 \%(95 \% \mathrm{CI}:-1.6 \%, 0.5 \%)$ respectively for the 32 and 16 studies (Figure 3, 4).

\section{Discussion}

Our results suggest that breastfeeding by $\mathrm{CHB}$ mothers does not pose a significant risk of infection by the virus, provided that the infants have been vaccinated against $\mathrm{HBV}$ at birth.

In this meta analysis, the overall transmission rate of HBV from CHB mothers to their infants who had completed routine hepatitis B vaccination was $4.32 \%$, which was similar to those previously reported [3]. Mother-toinfant transmission of HBV can occur prenatally, at birth and postnatally [49], but most commonly infection occurs at the time of delivery. In-utero transmission is very uncommon, except in China and Japan, and often persists after birth, which results in the ineffectiveness of HBV vaccination, i.e., immunoprophylaxis $[49,50]$. From this perspective, most mother-to-infant transmission occurs before deciding on infant feeding methods. But there is an exception: the neonate can be infected postnatally by household contact with $\mathrm{CHB}$ family members [51]. Apparently, breastfeeding would produce more frequent contacts between mother and infant than formula-feeding. Though separation of breastfed transmission of $\mathrm{CHB}$ from contact seems to be important in the clarification of the net effect of breastfeeding on infantile CHB infection, this contact transmission occurs only at the successful exchange of body fluid from infected mothers to their infants under some special situations, such as excoriation. Thus breastfed transmission of HBV, if existed, should be minimal.

Two previous meta analyses reported different associations of breastfeeding and infantile CHB infection with an odds ratio $(\mathrm{OR})=1.68(95 \% \mathrm{CI}: 1.16,2.44)[52]$ and 0.67 (95\%CI: 0.41, 1.12) [53] respectively based on 13 and 10 studies. The inconsistent results reflected difference between the studies with respect to their study aim, search strategies, criteria of inclusion and exclusion of the studies, and analysis strategies. One study included both vaccinated and not vaccinated infants [52] and the other included only those who had been given both passive and active immunization [53]. After careful evaluation on these two analyses, we identified their obvious shortcomings: (1) many eligible studies were not included in these analyses without any reason; (2) the analyses excluded those studies with zero outcome of infantile CHB infection in both groups of feeding methods; (3) neither of the studies evaluated the quality of the included studies and their influence on the results, and (4) neither of the studies considered the possible confounding of mothers' infectivity, which was important in mother-to-infant transmission of HBV and their decisions on how to feed their infants [17,54]. In our meta analysis, reference search, data extraction and quality evaluation were done by two independent

Table 2 Proportion of the mothers with high infectivity overall, by language and quality of papers

\begin{tabular}{lcccc}
\hline Groups & \multicolumn{2}{c}{ Breastfeeding } & \multicolumn{2}{c}{ Formula feeding } \\
\cline { 2 - 5 } & $\begin{array}{c}\text { No of high infectivity/ } \\
\text { Total No }\end{array}$ & $\begin{array}{c}\text { Proportion of } \\
\text { high infectivity (\%) }\end{array}$ & $\begin{array}{c}\text { No of high infectivity/ } \\
\text { Total No }\end{array}$ & $\begin{array}{c}\text { Proportion of } \\
\text { high infectivity (\%) }\end{array}$ \\
\hline All studies & $739 / 2001$ & 36.9 & $812 / 1843$ & 44.1 \\
Language of papers & & & & \\
$\quad \begin{array}{l}\text { English } \\
\quad \text { Chinese }\end{array}$ & $29 / 90$ & 32.2 & $60 / 239$ & 25.1 \\
Quality of papers & $710 / 1911$ & 37.2 & $752 / 1604$ & 46.9 \\
$\quad$ high & $37 / 109$ & & & $31 / 212$ \\
$\quad$ low & $702 / 1892$ & 33.9 & $741 / 1631$ & 43.5 \\
\hline
\end{tabular}

CHB: chronic hepatitis B infection 


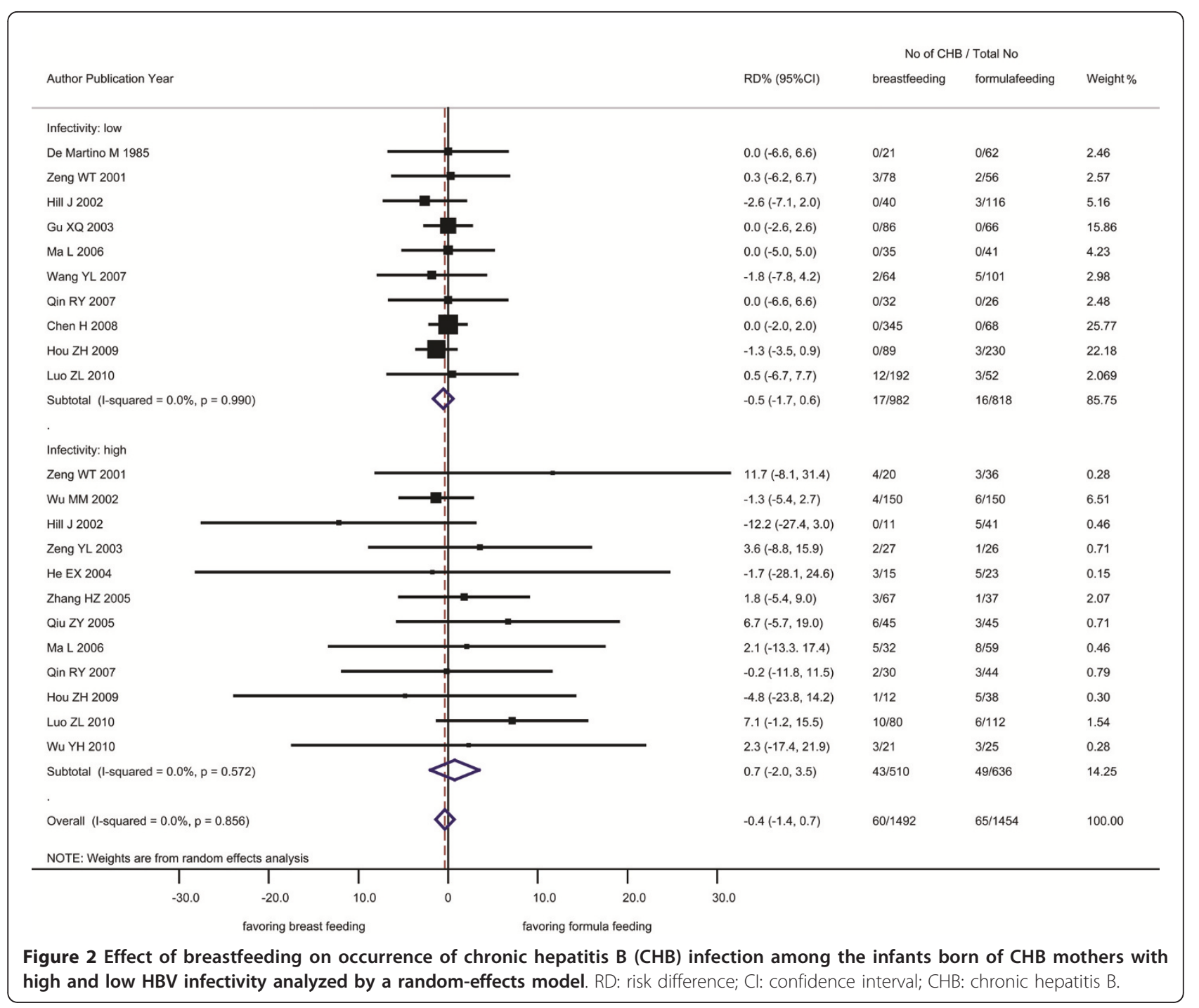

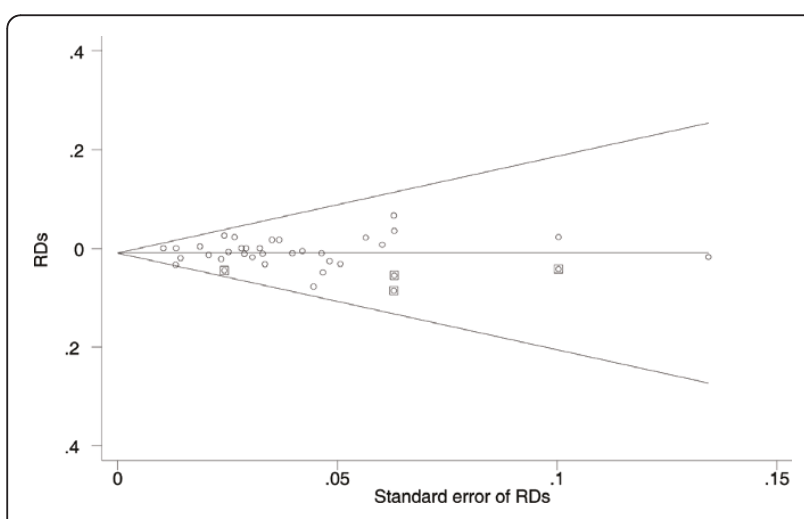

Figure 3 The Egger's funnel plot with pseudo $95 \%$ confidence limits for the 32 studies on the effect of breastfeeding on occurrence of chronic hepatitis B (CHB) infection among the infants born of CHB mothers. RD: risk difference; Cl: confidence interval; CHB: chronic hepatitis B. The circles alone and enclosed with square represented respectively the included and filled studies.

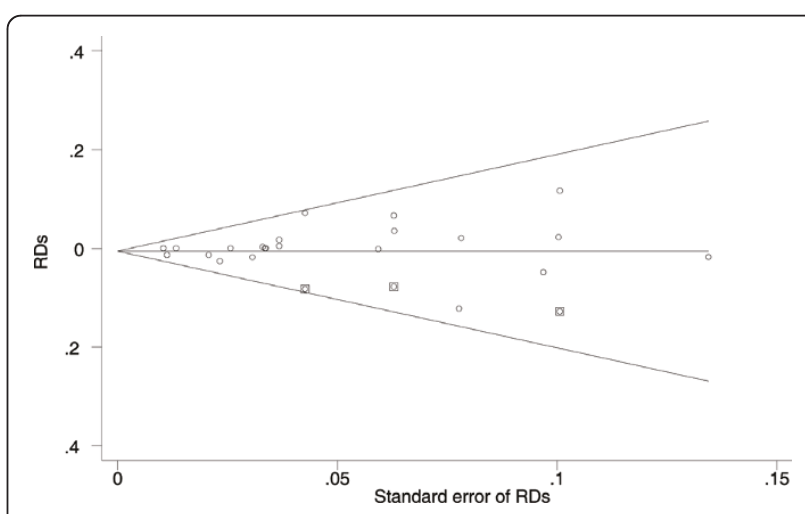

Figure 4 The Egger's funnel plot with pseudo $95 \%$ confidence limits for the 16 studies on the effect of breastfeeding on occurrence of chronic hepatitis B (CHB) infection among the infants born of CHB mothers with high and low infectivity. RD: risk difference; Cl: confidence interval; CHB: chronic hepatitis B. The circles alone and enclosed with square represented respectively the included and filled studies. 
investigators and examined by the third one before a consensus was reached, thus the completeness and appropriateness of the studies included for analysis were ensured. We used RD instead of OR or risk ratio (RR) as effect measure, which ensured that a reasonable estimation of effect measurement remained untouched and the $2^{\text {nd }}$ shortcoming mentioned above was overcome simultaneously.

Our findings have important implications for current clinical practice about breastfeeding by CHB mothers, even with high infectivity. Routine hepatitis $B$ vaccination allows the infant to enjoy breastfeeding, the nutritional, immunological and psychological advantages of which are well known[4]. And breastfeeding would also greatly benefit the mother[4], especially in weakening or eliminating her fear and guilt which occur often. Unfortunately, contrary to our findings that risk of breastfed transmission of infantile CHB infection simply does not exist, the current clinical practices seem discouraging: (1) any breastfeeding rate is about $30 \%$ lower for those mothers with than without CHB [54], and even lower (5.4\% only) for those with high infectivity [55]; (2) there is still $25 \%-50 \%$ of the medical professionals did not recommend infantile breastfeeding by $\mathrm{CHB}$ mothers $[15,16]$. Thus, health education of breastfeeding with correct knowledge, attitude and behaviour on the decision makers, such as medical professional and CHB mothers, seems to be urgent, especially in those epidemic countries, like China.

This meta analysis demonstrates no association between breastfeeding and risk of infantile CHB infection from a CHB mother to her infant. Two alternative explanations must be considered. First, a systematic bias may be present in follow-up studies arising from differential lost to follow-up, which has not been mentioned in most of the studies. Second, the studied population varies from study to study, and some unknown confounders may not be effectively evaluated. Further evaluation of the effect of breastfeeding from an HBV infected mother and infantile CHB infection while taking into consideration of potential biases can be feasibly achieved with more large-scale prospective follow-up studies.

\section{Conclusions}

Breast milk is infectious; yet, breastfeeding, even by mothers with high infectivity, is not associated with demonstrable risk of infection of infantile CHB. This may be partly because infection mainly occurs during delivery and the protection by vaccination. The finding concurs with that of individual studies and supports the WHO recommendation of $\mathrm{BF}$, irrespective of the $\mathrm{HBV}$ status of the mothers.

\section{Additional material}

Additional file 1: Figure S1 Flow chart for the process of retrieving papers in our meta analysis. CBM: Chinese BioMedical Literature database, CNKI: Chinese National Knowledge Infrastructure.

\section{Acknowledgements}

This work was supported by a grant from the Ministry of Science and Technology of the People's Republic of China (2008ZX10002-012) and the Shanghai Leading Academic Discipline Project (B118)

I thank Professor Munhon Ng for his critical reading of this manuscript, the reviewers for their helpful comments and Mr Peter Stephen Liu for English editing.

\section{Author details}

'Department of Epidemiology, School of Public Health, Fudan University, Shanghai 200032, China. ${ }^{2}$ The Key Laboratory on Public Health Safety, Ministry of Education, Fudan University, Shanghai 200032, China. ${ }^{3}$ Library of Medical Centre, Fudan University, Shanghai 200032, China.

\section{Authors' contributions}

YJZ conceived, coordinated the study and drafted the manuscript. YL participated in the design and data analysis. QY and YQZ participated in the design, reference search and data extraction. YX participated in quality rating and data analysis. QQY and SW participated in quality rating, data extraction and analysis. All authors read and approved the final manuscript.

\section{Competing interests}

The authors declare that they have no competing interests.

Received: 3 March 2011 Accepted: 27 June 2011

Published: 27 June 2011

\section{References}

1. Lavanchy D: Hepatitis B virus epidemiology, disease burden, treatment, and current and emerging prevention and control measures. J Viral Hepat 2004, 11(2):97-107

2. Lok AS: Chronic hepatitis B. N Engl J Med 2002, 346(22):1682-1683.

3. Lee CY, Huang LM, Chang MH, Hsu CY, Wu SJ, Sung JL, Safary A: The protective efficacy of recombinant hepatitis $B$ vaccine in newborn infants of hepatitis B e antigen-positive-hepatitis B surface antigen carrier mothers. Pediatr Infect Dis J 1991, 10(4):299-303.

4. Gartner LM, Morton J, Lawrence RA, Naylor AJ, O'Hare D, Schanler RJ, Eidelman Al: Breastfeeding and the use of human milk. Pediatrics 2005, 115(2):496-506.

5. Linnemann CC, Goldberg S: HBAg in breast milk. Lancet 1974, , 2: 155.

6. Petrova M, Kamburov V: Breastfeeding and chronic HBV infection: clinical and social implications. World J Gastroenterol 2010, 16(40):5042-5046.

7. Ma L, Zhao GZ, Liang ZL: Relationship between load of HBV-DNA in serum and breast milk for pregnant women and safety of breastfeeding. China Journal of Modern Medicine 2006, 16(17):2581-2585.

8. Beasley RP, Stevens CE, Shiao IS, Meng HC: Evidence against breastfeeding as a mechanism for vertical transmission of Hepatitis B. Lancet 1975, 2(7938):740-741.

9. Derso A, Boxall E, Tarlow M, Flewett T: Transmission of HBsAg from mother to infant in four ethnic groups. Br Med J 1978, 1(6118):949-952.

10. Hill J, Sheffield J, Kim M, Alexander J, Sercely B, Wendel G: Risk of hepatitis B transmission in breast-fed infants of chronic hepatitis B carriers. Obstet Gynecol 2002, 99(6):1049-1052.

11. De Martino M, Appendino C, Resti M, Rossi ME, Muccioli AT, Vierucci A: Should hepatitis B surface antigen positive mothers breastfeed? Arch Dis Child 1985, 60:972-974.

12. Tseng RY, Lam CW, Tam J: Breastfeeding babies of HBsAg-positive mothers. Lancet 1988, 2(8618):1032.

13. Wang JS, Zhu QR, Wang XH: Breastfeeding does not pose any additional risk of immunoprophylaxis failure on infants of HBV carrier mothers. Int J Clin Pract 2003, 57(2):100-102. 
14. World Health Organization: Hepatitis B and breastfeeding. J Int AssoC Physicians AIDS Care 1998, 4(7):20-21.

15. Giles ML, Garland SM, Grover SR, Lewin SM, Hellard ME: Impact of an education campaign on management in pregnancy of women infected with a blood-borne virus. Med J Aust 2006, 184(8):389-392.

16. Ahn J, Salem SB, Cohen SM: Evaluation and management of hepatitis B in pregnancy: a survey of current practices. Gastroenterol Hepatol (NY) 2010, 6(9):570-578.

17. Lee C, Gong Y, Brok J, Boxall EH, Gluud C: Effect of hepatitis B immunisation in newborn infants of mothers positive for hepatitis $B$ surface antigen: systematic review and meta-analysis. BMJ 2006, 332(7537):328-336.

18. Wells G, Shea BO, Connell D: The Newcastle-Ottawa Scale (NOS) for assessing the quality of nonrandomized studies in meta-analysis. 2010 [http://www.ohri.ca/programs/_linical_epidemiology/oxford_web.ppt].

19. Altman $\mathrm{DG}$, Bland JM: Interaction revisited: the difference between two estimates. BMJ 2003, 326(7382):219.

20. Begg CB, Mazumdar M: Operating characteristics of a rank correlation test for publication bias. Biometrics 1994, 50(4):1088-1101.

21. Duval S, Tweedie R: A Nonparametric "Trim and Fill" Method of Accounting for Publication Bias in Meta-Analysis. J Am Stat Assoc 2000, 95(449):89-98.

22. Huang YR, Guo $\mathrm{CJ}$, Huang GH: Breastfeeding among HBsAg positive mothers: a report of 126 cases. The Journal of Clinical Pediatrics 1993, 11(5):331-332.

23. Wu MM, Su PM: A primary study on the breastfeeding among HBsAg positive mothers. Jiangsu Medical Journal 1996, 22:410-412.

24. Zeng WT, Zhu KL, Ma PQ, Deng ZX, Wei JJ, Ye XM: Clinical study on the relationship between breastfeeding and $\mathrm{HBV}$ transmission. Chinese Journal of Integrated Traditional and Western Medicine on Liver Diseases 2001, 11(1):13-14.

25. Wu MM, Tang YR, Guo CX, Fang ZX: Observation on the results of breastfeeding of hepatitis $B$ carrier mothers after using active and passive immunization. Chinese Journal of Obstetrics and Gynecology 2002, 4: $235-236$.

26. Zeng $Y L$, Wu J, Wang L, Hu R, Pu HM, Zhu LX: The safety of breastfeeding among the mothers positive in serum indices of hepatitis $B$ virus. Sichuan Medical Journal 2003, 24(8):823-824.

27. Gu XQ, Shi LE, Han SP, Wu B, Sun HJ: Interruption of mother-to-infant transmission of hepatitis $B$ virus and comparison of immunological effect of recombinant and blood derived hepatitis B vaccine. Journal of Applied Clinical Pediatrics 2003, 18(11):895-897.

28. He EX, Jiang L: The effect of breastfeeding on the infants borne of the mothers with hepatitis B. Inner Mongolia Medical Journal 2004, 36(5):37.

29. Liu DM, Wang XX: Clinical study on the transmission of heapatitis $B$ virus through breast milk after combined active and passive immuneprophylaxis. Journal of Medical Reaserch 2004, 22(4):56-57.

30. Yao WQ, Li DH, Chen Y, Huang XD: The safety of breastfeeding among HBV carrier mothers after combined active and passive immunoprophylaxis. Chinese Journal of Practical Gynecology and Obstetrics 2004, 20(2):109-110.

31. Meng Z, Xiao XM, He MJ, Xu YY, Li Y: The application of HBIG to maternal women carring $\mathrm{HBsAg}$ and the effect of different feeding way to infants. The Journal of Practical Medicine 2004, 20(10):1102-1104.

32. Mou RL, Ma YY, Li H, Kong BH, Jiang S: Study on breastfeeding in hepatitis B virus carrier women. Chinese Journal of Perinatal Medicine 2005, 8(1):20-23.

33. Zhang $H Z$, Ma MX, Li X, Tian MQ: The effect of mother-to-infant transmission of hepatitis $B$ through hepatitis $B$ immunization among 304 cases. Chinese Journal of Integrated Traditional and Western Medicine on Liver Diseases 2005, 15(5):289-290.

34. Zhang JH, Wang $F L, H u Y, X u B$, Ding $A J$, Jiang $L$ : An investigation on the safety of breastfeeding among infants born of hepatitis-B-virus-markerspositive mothers by active and passive immunization. Journal of Chongqing Medical University 2005, 30(2):275-277.

35. Qiu ZY: Investigation on breastfeeding of 90 maternal mothers with positive HBeAg. Zhejiang Journal of Preventive Medicine 2005, 17(9):39-44.

36. Zeng DY, Mo KL, He HY, Guan J, Fang ZY, Zhong H: Risk of HBV transmission in breast-fed infants of chronic HBV carriers after combined active and passive immunoprophylaxis. Progress in Obstetrics and Gynecology 2006, 15(2):125-127.
37. Chen JY: The investigation on breastfeeding of the mothers with hepatitis B virus. Practical Clinical Journal of Integrated Traditional Chinese and Western Medicine 2006, 6(2):66-67.

38. Zuo SE, Ma YL, Zhang QT, Wang LJ, Qiang GY: The safety of breastfeeding among HBV carriers after hepatitis B immunization. Maternal and Child Health Care of China 2007, 22(13):1734-1735.

39. Wang $Y L$, Gu J, Zhao L: The relationship between breastfeeding and incidence of mother-to-infant transmission of hepatitis B virus: a report of 165 cases. Ningxia Medical Journal 2007, 29(5):415-416.

40. Qin RY, Shen BS: A clinical study on the safety of breastfeeding after immunoprophylaxis among positive HBsAg mothers. Maternal and Child Health Care of China 2007, 22(31):4468-4469.

41. Chen H, Han J, Yang HS: A contrast study on influence of different feeding and immunity patterns of $\mathrm{HBsAg}$ carriers on HBV horizontal transmission to their infants. Chinese Journal of Woman and Child Health Research 2008, 19(4):316-318.

42. Hou ZH, Yu WH, Zhou XM, Wang XL: Risk of vertical transmission through breastfeeding among chronic hepatitis B infection. International Journal of Laboratory Medicine 2009, , 12: 1238-1239.

43. Li Y: The safety study of breastfeeding among hepatitis B virus carrier mothers. Chinese Community Doctors 2009, 5: 16-17.

44. Luo ZL, Zheng X: Study on the relationship between breastfeeding of HBsAg positive mothers and the transmission of hepatitis $B$ virus to their infants. Fujian Medical Journal 2010, 32(5):45-47.

45. Chen FJ, Qi ZB, Wang J: Impact of HBV-DNA levels on the blocking of transmission of hepatitis $B$ from mothers to babies by injection of hepatitis B immunoglobulin. China Tropical Medicine 2010, 10(12):45-47.

46. Sun $L Y$, Zhao ZM, Liu C: The safety of breastfeeding by the mothers with HBV infection. China Modern Doctor 2010, 48(3):138-139.

47. Liu GX, Zheng AH, Chen SN: Safety of breastfeeding in infants of HBV carrier mothers after combined active and passive immunoprophylaxis in both mothers and infants. Chinese Journal of Modern Drug Application 2010, 4(12):9-10.

48. Wu YH: Serum HBV-DNA level and safety of breastfeeding. China Modern Doctor 2010, 48(29):89-90.

49. Ranger-Rogez S, Denis F: Hepatitis B mother-to-child transmission. Expert Rev Anti Infect Ther 2004, 2(1):133-145.

50. Wang Z, Zhang J, Yang H, Li X, Wen S, Guo Y, Sun J, Hou J: Quantitative analysis of HBV DNA level and HBeAg titer in hepatitis B surface antigen positive mothers and their babies: HBeAg passage through the placenta and the rate of decay in babies. J Med Virol 2003, 71(3):360-366.

51. Ordog K, Szendroi A, Szarka K, Kugler Z, Csire M, Kapusinszky B, Xie J, Csizmadia K, Brojnas J, Rusvai E, Tempfli A, Berencsi G: Perinatal and intrafamily transmission of hepatitis $B$ virus in three generations of a low-prevalence population. J Med Virol 2003, 70(2):194-204.

52. Tang XM, Xiao XM: Meta-analysis on the safety of breastfeeding on the infants of HBV carrier mothers. Maternal and Child Health Care of China 2007, 22(29):4193-4195.

53. Liu ZQ: The effect of feeding methods on infant transmission of hepatitis B virus after combined immunoprophylaxis: a meta analysis. Zhejiang University; 2009.

54. Qiu L, Binns CW, Zhao Y, Zhang K, Xie X: Hepatitis $B$ and breastfeeding in Hangzhou, Zhejiang Province, People's Republic of China. Breastfeed Med 2010, 5(3):109-112

55. Guo Y, Liu J, Meng L, Meina H, Du Y: Survey of HBsAg-positive pregnant women and their infants regarding measures to prevent maternalinfantile transmission. BMC Infect Dis 2010, 10:26.

\section{Pre-publication history}

The pre-publication history for this paper can be accessed here: http://www.biomedcentral.com/1471-2458/11/502/prepub

doi:10.1186/1471-2458-11-502

Cite this article as: Zheng et al:: Should chronic hepatitis B mothers breastfeed? a meta analysis. BMC Public Health 2011 11:502. 